\title{
Focus Point on Tests of General Relativity and Alternative Gravity Theories
}

Published online: 3 June 2019 - C Società Italiana di Fisica / Springer-Verlag GmbH Germany, part of Springer Nature, 2019 If an experiment agrees with a theory it means for the latter "Maybe", and if it does not agree it means "No".
Probably every theory will someday experience its "No" - most theories, soon after conception.

A. Einstein (1922)

Testing of fundamental physical theories at ever higher accuracy is a continuous process and hence General Relativity and the development of alternative gravity theories have always been among the interests of experimentalists, astronomers and theoreticians. The empirical basis of General Relativity is linked to an immense scope of areas, from the equivalence principle up to, say, the variation of the gravitational constant and of other physical constants.

In 1960s the General Relativity was transferred from abstract mathematical construction to a driving force in a new area of research - relativistic astrophysics - dealing with a sequence of impressive observational discoveries, such as quasars, cosmic microwave background radiation, pulsars, compact binary stars, etc. Practically at the same time, the development of rockets and space technologies provided means for new types of tests of General Relativity, such as Lunar laser ranging and signal time delays. Let us recall a remark attributed to Einstein at the end of a discussion with Thirring in 1918 in regard to a test of frame-dragging: What a pity the earth has no moon in an orbit just outside its atmosphere! Currently, laser-ranged satellites are among the important tools for testing tiny predictions of General Relativity, such as frame-dragging. Some alternative gravitational theories agree with all the current tests of General Relativity but predict different results for frame-dragging.

A new phase of studies on extensions of General Relativity and alternative gravity theories started with the discovery of the dark sector - dark energy and dark matter - as of dominating content of the Universe. Cosmology, therefore, became an area of broad applications of alternative gravity theories. Informative constraints on gravity theories are also obtained monitoring S2 and other stars in the vicinity of the massive black hole in the Galactic center. Thus, it is now hardly possible to mention cosmic structures, from the Solar system up to the dynamics of galaxies and clusters of galaxies, for which alternative gravity theories have not been probed.

The discovery of gravitational waves, one hundred years after the formulation of General Relativity, further stimulated the consideration of alternative gravity theories. The particular importance of this new window is in that it enables to test the strong-field General Relativity, i.e. the effects close to the event horizon.

Finally, testing the Equivalence Principle is a probe for gravitational theories at fundamental level and a main step towards any quantum gravity theory.

The papers included in this Focus Point naturally cover a minor fraction of the above-mentioned areas; nevertheless they contain timely accounts on broad topics, from tests on the Equivalence Principle, to orbital dynamics of extended bodies in inspiraling binary systems, up to cosmology. 